\title{
Identification of novel long non-coding RNAs in clear cell renal cell carcinoma
}

\author{
Jasmine JC Blondeau', Mario Deng ${ }^{2,3,4}$, Isabella Syring ${ }^{1,3}$, Sarah Schrödter ${ }^{1}$, Doris Schmidt', Sven Perner ${ }^{2,3,4}$, \\ Stefan C Müller ${ }^{1}$ and Jörg Ellinger ${ }^{1 *}$
}

\begin{abstract}
Background: Long non-coding RNAs (IncRNA) play an important role in carcinogenesis; knowledge on IncRNA expression in renal cell carcinoma is rudimental. As a basis for biomarker development, we aimed to explore the IncRNA expression profile in clear cell renal cell carcinoma (ccRCC) tissue.

Results: Microarray experiments were performed to determine the expression of 32,183 IncRNA transcripts belonging to 17,512 IncRNAs in 15 corresponding normal and malignant renal tissues. Validation was performed using quantitative real-time PCR in 55 cCRCC and 52 normal renal specimens. Computational analysis was performed to determine IncRNA-microRNA (MiRTarget2) and IncRNA-protein (catRAPID omics) interactions. We identified 1,308 dysregulated transcripts (expression change >2-fold; upregulated: 568, downregulated: 740) in cCRCC tissue. Among these, aberrant expression was validated using PCR: Inc-BMP2-2 (mean expression change: 37-fold), Inc-CPN2-1 (13-fold), Inc-FZD1-2 (9-fold), Inc-ITPR2-3 (15-fold), Inc-SLC30A4-1 (15-fold), and Inc-SPAM1-6 (10-fold) were highly overexpressed in cCRCC, whereas Inc-ACACA-1 (135-fold), Inc-FOXG1-2 (19-fold), Inc-LCP2-2 (2-fold), Inc-RP3-368B9 (19-fold), and Inc-TTC34-3 (314-fold) were downregulated. There was no correlation between IncRNA expression with clinical-pathological parameters. Computational analyses revealed that these IncRNAs are involved in RNA-protein networks related to splicing, binding, transport, localization, and processing of RNA. Small interfering RNA (siRNA)-mediated knockdown of Inc-BMP2-2 and Inc-CPN2-1 did not influence cell proliferation.
\end{abstract}

Conclusions: We identified many novel IncRNA transcripts dysregulated in ccRCC which may be useful for novel diagnostic biomarkers.

\section{Background}

Renal cell carcinoma (RCC) is one of the most common malignancies; its incidence is varying substantially worldwide: RCC incidence is high in Europe and North America and low in Asia and South America [1]. Today, many small-sized renal tumors are diagnosed; however, imaging modalities do not allow precise differentiation between renal cell carcinoma and non-malignant renal tumors. Performing tumor biopsy and histopathological classification is sometimes challenging, and definitive exclusion of malignancies still requires surgical exploration. As active surveillance protocols for small renal lesions find the way into daily clinical practice, a better estimation of tumor aggressiveness becomes

\footnotetext{
* Correspondence: joerg.ellinger@ukb.uni-bonn.de

'Department of Urology, University Hospital Bonn, Bonn, Germany

Full list of author information is available at the end of the article
}

necessary. Thus, identifying novel biomarkers would be helpful for the management of patients with renal tumors.

Nucleic acids are under discussion as potential biomarkers for patients with RCC [2]. Long non-coding RNAs (lncRNA) are a class of RNA molecules arbitrarily defined as being longer than 200 nucleotides and not translated into a protein. Initially, it was thought that lncRNAs represent transcriptional noise, but it is nowadays recognized that lncRNAs may have biological roles. They are regulating imprinting, dosage compensation, cell cycle, pluripotency, retrotransposon silencing, and meiotic entry, for example [3]. However, knowledge on lncRNA expression and their function is still in its infancy, but lncRNA expression seems to be highly tissue and tumor specific [4]. So far, few are known on lncRNAs expression in RCC [5-8]. We therefore 
performed microarray experiments to study the expression of 32,183 lncRNA transcripts in a cohort of patients with clear cell RCC (ccRCC) to determine a comprehensive lncRNA profile.

\section{Results}

Microarray: screening for aberrantly expressed IncRNAs A gene expression microarray was used to identify dysregulated lncRNAs in 15 corresponding tumor and normal renal tissue samples as a discovery cohort. Among the 32,183 analyzed lncRNA transcripts, we observed differential expression (fold change >2) in 1,308 transcripts: 568 IncRNA transcripts were upregulated and 740 were downregulated in ccRCC samples. The 20 most differentially expressed lncRNAs in ccRCC and normal renal tissue are listed in Table 1. A hierarchical cluster analysis based on centered Pearson correlation coefficient was performed to determine different expression profiles in ccRCC and normal tissue. As shown in Figure 1, the lncRNA expression profile allowed distinguishing cancerous and normal tissue samples highly accurately.

\begin{tabular}{|c|c|c|c|}
\hline \multicolumn{2}{|c|}{ Downregulated in cancer } & \multicolumn{2}{|c|}{ Upregulated in cancer } \\
\hline IncRNA & $\begin{array}{l}\text { Log2 fold } \\
\text { change }\end{array}$ & IncRNA & $\begin{array}{l}\text { Log2 fold } \\
\text { change }\end{array}$ \\
\hline Inc-TTC34-3 & -6.585 & Inc-CPN2-1 & 5.966 \\
\hline Inc-ACACA-1 & -6.486 & Inc-BMP2-2 & 4.787 \\
\hline Inc-LCP2-2 & -5.771 & Inc-DGCR6-2 & 4.659 \\
\hline Inc-AC068473.1-2 & -5.654 & Inc-ITPR2-3 & 4.379 \\
\hline Inc-MED10-7 & -5.145 & Inc-SLC30A4-1 & 4.150 \\
\hline Inc-TMEM18-8 & -4.880 & Inc-NR2E1-1 & 4.131 \\
\hline Inc-ATP1A1OS-2 & -4.770 & Inc-FZD1-2 & 4.019 \\
\hline Inc-FOXG1-2 & -4.485 & Inc-DGCR6-2 & 3.920 \\
\hline Inc-TRAT1-2 & -4.484 & Inc-PADI1-1 & 3.904 \\
\hline Inc-RP3-368B9.1.1-1 & -4.453 & Inc-SPAM1-6 & 3.516 \\
\hline Inc-IFRD2-1 & -4.440 & Inc-PRRC2C-1 & 3.508 \\
\hline Inc-TBCCD1-1 & -4.369 & Inc-MYC-2 & 3.483 \\
\hline Inc-C15orf2-2 & -3.981 & Inc-CDRT15L2-1 & 3.479 \\
\hline Inc-ATP8A1-1 & -3.960 & Inc-SCRG1-1 & 3.275 \\
\hline Inc-LY86-3 & -3.876 & Inc-TMEM30B-5 & 3.260 \\
\hline Inc-SCN2A-2 & -3.787 & Inc-RCBTB2-1 & 3.205 \\
\hline Inc-ST6GALNAC3-1 & -3.786 & Inc-AHR-1 & 3.034 \\
\hline Inc-MIS18BP1-1 & -3.557 & Inc-DLX2-4 & 2.903 \\
\hline Inc-RASL11B-2 & -3.532 & Inc-HPS3-2 & 2.720 \\
\hline Inc-RACGAP1-2 & -3.528 & Inc-RCBTB2-3 & 2,709 \\
\hline
\end{tabular}

\section{Real-time PCR: validation of expression profiling}

In order to confirm aberrant lncRNA expression, we determined the expression of 13 lncRNAs in an independent validation cohort of ccRCC $(n=55)$ and normal $(n=52)$ renal tissue samples. The selection of representative IncRNAs for validation was based on the degree of dysregulation in the microarray (six upregulated and five downregulated lncRNA; in addition two non-regulated lncNRA). As expected, lnc-BMP2-2 (mean: 37-fold), lncCPN2-1 (13-fold), lnc-FZD1-2 (9-fold), lnc-ITPR2-3 (15-fold), lnc-SLC30A4-1 (15-fold), and lnc-SPAM1-6 (10fold; all $p<0.001$ ) were highly overexpressed in RCC, whereas lnc-ACACA-1 (135-fold), lnc-FOXG1-2 (19-fold), lnc-LCP2-2 (2-fold), lnc-RP3-368B9 (19-fold), and lncTTC34-3 (314-fold) were downregulated (all $p<0.001$ ). Both, lnc-ERCC5-1 $(p=0.401)$ and lnc-RP11-480I12.4.1-1 $(p=0.731)$ were-as observed in the microarray studiesnot dysregulated in tumor samples. The lncRNA expression levels allowed highly sensitive and specific discrimination between RCC and control subjects as determined by using ROC analyses: the area under the curve was $>0.9$ for all dysregulated samples, especially lnc-CPN2-1 overexpression allowed molecular identification of RCC tissue (AUC 0.942, 95\% confidence interval 0.884-1.000). See Figure 2 and Table 2.

We also analyzed whether lncRNA expression levels were correlated with poor prognostic parameters; the Bonferroni method was applied to correct for multiple hypothesis testing (adjusted significance value $p<0.0083$ ). None of the lncRNAs was significantly correlated with staging nor grading. However, there was a tendency towards lower lnc-ERCC5-1 $(p=0.034)$ and RP3-368B9 $(p=0.016)$ levels in Fuhrman grade 3/4 tumors, lower lnc-ERCC5-1 $(p=0.026)$ expression in vascular invasive tumors, and advanced AJCC stage in samples with low lnc-RP3-368B9 $(p=0.011)$. IncRNAs were not associated with progression-free survival, overall survival or cancer-specific survival (all $\mathrm{p}>0.05$, data not shown), as determined using Cox regression analysis.

\section{siRNA-mediated IncRNA knockdown}

We next treated Caki-1, Caki-2, and A-498 RCC cell lines with small interfering RNA (siRNA) targeting lncBMP2-2 and lnc-CPN2-1; see Figure 3. lnc-BMP2-2 expression was significantly $(p<0.05)$ decreased in siRNA-treated Caki-1 and Caki-2 (only siRNA \#2) cells; it was basically unexpressed in A-498 cells. lnc-CPN2-1 was also decreased in Caki-2 cells $(p<0.05)$. Glyceraldehyde 3-phosphate dehydrogenase (GAPDH) expression was significantly reduced in all cell lines (not shown); the negative controls did not change gene expression. However, the EZ4U test did not show any changes in cellular proliferative activity after siRNA-mediated lncRNA knockdown. See Figure 4. 


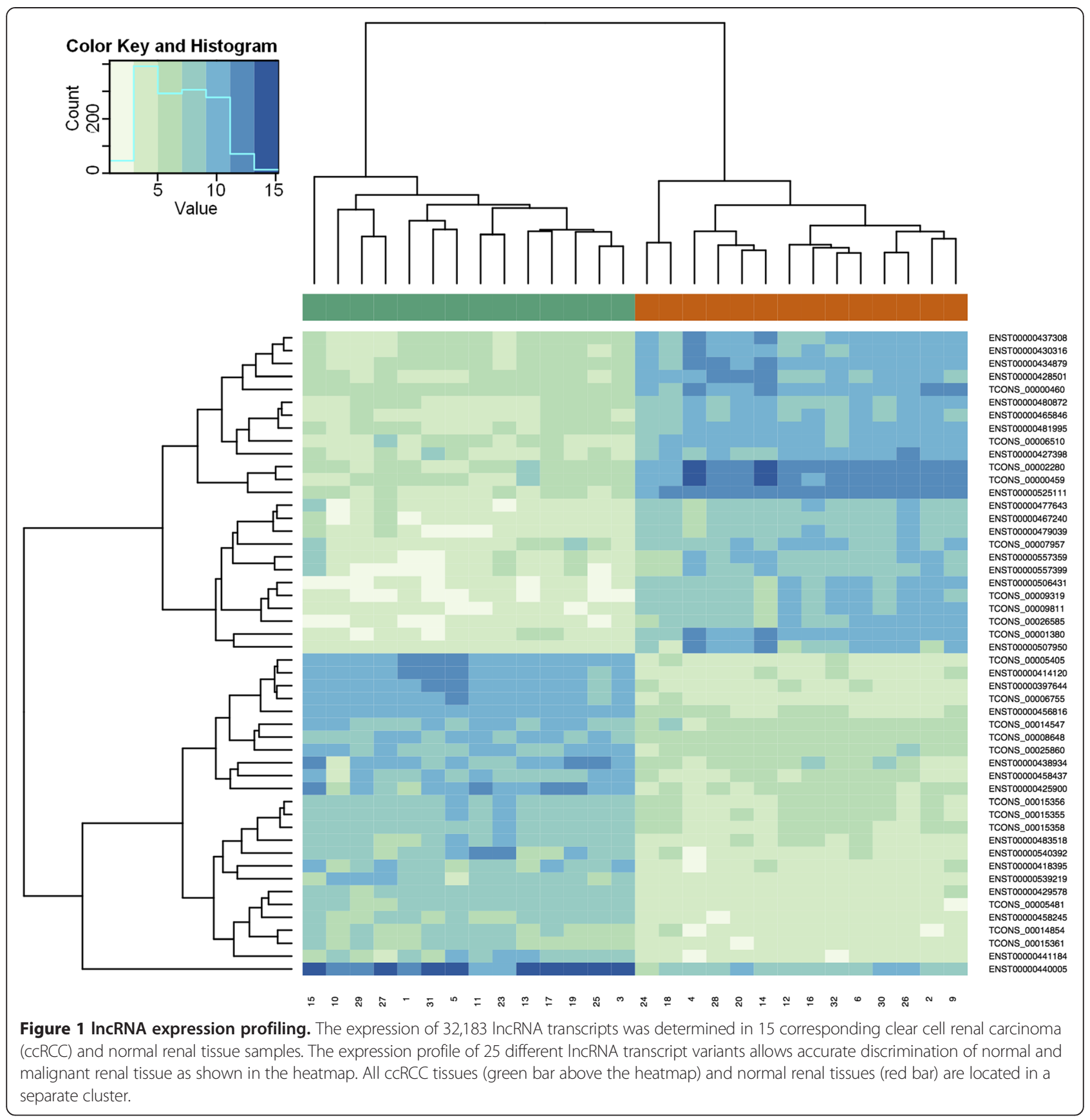

\section{Computational prediction of microRNA targeting and protein interaction}

The MiRTarget2 algorithm [9] was used to predict microRNA seeds within the validated lncRNA transcripts. Most transcripts do not have any (lnc-BMP2-2; lnc-FZD1-2; lnc-ITPR2-3:1; lnc-SPAM1-6; lnc-TTC34-4) or only few (lnc-ACACA-1: miR-4652-3p, miR-5001-3p, miR-47533p; lnc-CPN2-1: miR-149-3p; lnc-LCP2-2: miR-3191-5p; lnc-SLC30A4-1: miR-4772-5p) predicted targeting microRNAs with-so far-unknown functions. lnc-FOXG1-2 has multiple predicted target microRNAs (miR-3662, miR-3120-5p, miR-519c-3p, miR-519a-3p, miR-548ag, miR-4282, miR-519b-3p, miR-458 m, miR-3163, miR4658, miR-676-5p, miR-3973). It was reported that lncRNAs can act as a microRNA sponge by binding specific microRNAs and thereby by interfering with their role as regulator of gene expression [10]. However, none of the predicted microRNA targets of FOXG1-2 was correlated to FOXG1-2 levels in a cohort of each ten normal and malignant renal tissue samples (data not shown; miR- 

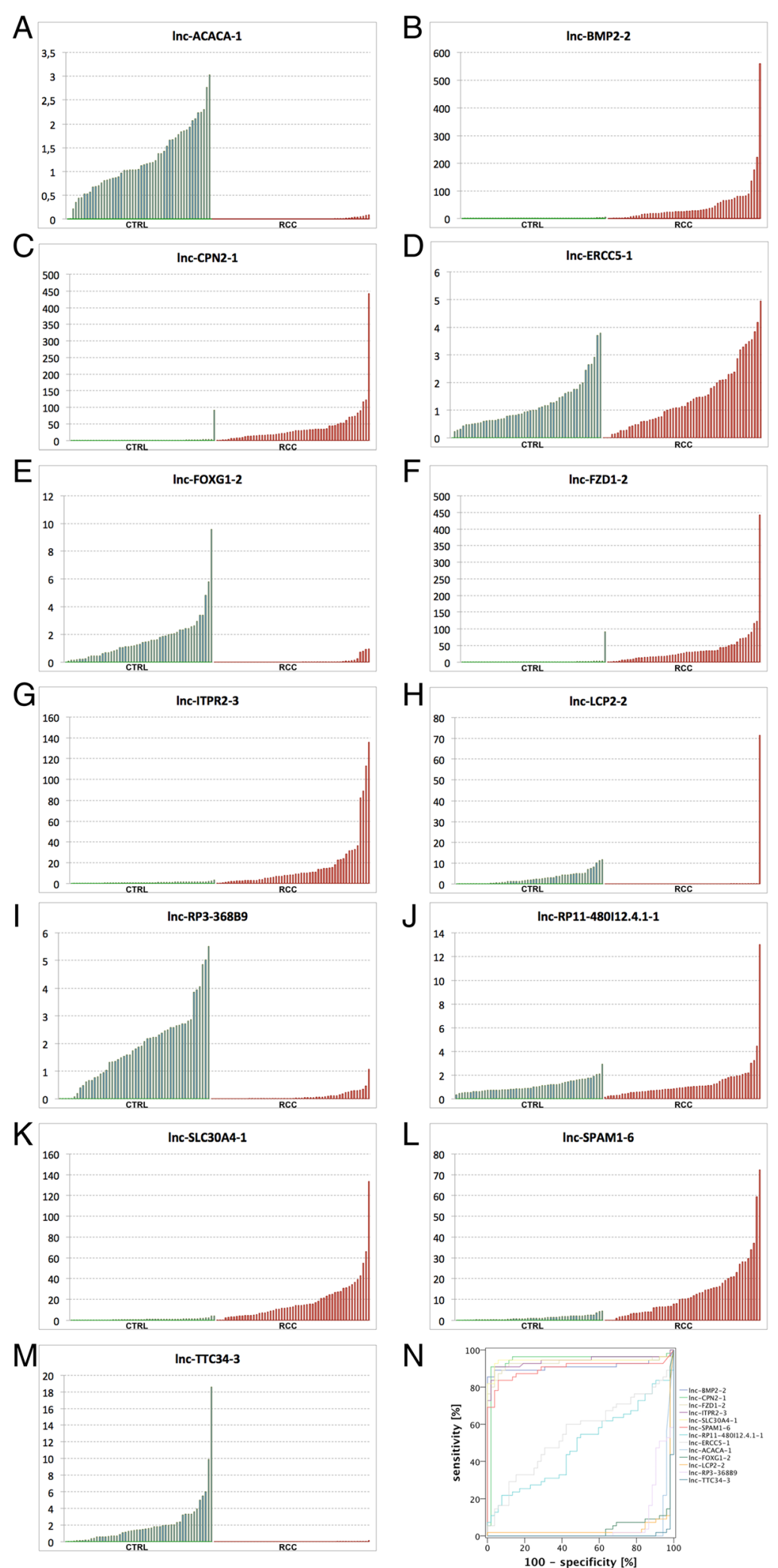

Figure $\mathbf{2}$ (See legend on next page.) 
(See figure on previous page.)

Figure 2 Validation of IncRNA dysregulation. The expression of 13 target IncRNAs in renal cell carcinoma (red) and normal renal (green) tissue was validated using quantitative real-time PCR; the expression levels were normalized using ACTB and PPIA as reference genes. We confirmed significant (all $p<0.001$ ) overexpression of Inc-BMP2-2 (B), Inc-CPN2-1 (C), Inc-FZD1-2 (F), Inc-ITPR2-3 (G), Inc-SLC30A6-1 (K), and Inc-SPAM1-6 (L) in renal cell carcinoma, whereas Inc-ACACA-1 (A), Inc-FOXG1-2 (E), Inc-LCP2-2 (H), Inc-RP3-368B9 (I), and Inc-TTC34-3 (M) were significantly downregulated (all $p<0.001$ ); Inc-ERCC5-1 (D, $p=0.401)$ and Inc-RP11-480112.4.1-1 (J, $p=0.731$ ) were not different in malignant and normal renal tissue. Receiver operator characteristic $\mathbf{( N )}$ analyses demonstrate excellent discrimination of the IncRNAs between both cohorts (area under the curve $0.90-0.94)$.

519a, miR-519b, miR-519c, miR-548, miR-3120, miR4284, miR-4658: all $p>0.1$ ). MicroRNA expression was similar in RCC and normal renal tissue (all $p>0.5$ ).

Furthermore, we determined lncRNA-protein interactions using the catRAPID omics algorithm [11]. Numerous lncRNA-protein interactions $(n=91)$ are predicted for all transcripts (see Additional file 1: Table S2); the cellular functions of proteins with a ranking score $>2.5$ were next explored using GeneMANIA [12]. The interacting networks are mainly related to splicing, binding, transport, localization, and processing of RNA but also transcription and translation (see Table 3; Additional file 2: Table S3).

\section{Discussion}

lncRNA are important regulators of gene expression during genetic information processing in living cells and interact with major cellular pathways (e.g. proliferation, differentiation, apoptosis). Thereby, they are involved in carcinogenesis of many human malignancies [13], but so far, knowledge about lncRNA expression in RCC is limited [5-8]. We determined the expression of 32,183 lncRNA transcripts belonging to 17,512 lncRNAs in 15 corresponding normal and malignant renal tissues, thereby providing the most comprehensive analysis of

Table 2 Receiver operator characteristic analyses for discrimination of normal and malignant renal tissue based on IncRNA expression

\begin{tabular}{lll}
\hline IncRNA & $\begin{array}{l}\text { Area under } \\
\text { the curve }\end{array}$ & $\begin{array}{l}\mathbf{9 5 \%} \text { confidence } \\
\text { interval }\end{array}$ \\
\hline Inc-FZD1-2 upregulation & 0.931 & $0.871-0.991$ \\
Inc-SLC30A4-1 upregulation & 0.942 & $0.883-1.000$ \\
Inc-BMP2-2 upregulation & 0.912 & $0.843-0.981$ \\
Inc-SPAM1-6 upregulation & 0.900 & $0.830-0.969$ \\
Inc-ITPR2-3 upregulation & 0.941 & $0.887-0.994$ \\
Inc-CPN2-1 upregulation & 0.942 & $0.884-1.000$ \\
Inc-TTC34-3 downregulation & 0.990 & $0.973-1.000$ \\
Inc-ACACA-1 downregulation & 0.966 & $0.923-1.000$ \\
Inc-LCP2-2 downregulation & 0.955 & $0.906-1.000$ \\
Inc-FOXG1-2 downregulation & 0.954 & $0.911-0.997$ \\
Inc-RP3-368B9.1.1-1 downregulation & 0.938 & $0.892-0.984$ \\
\hline
\end{tabular}

lncRNA expression in RCC up to now. We observed differential expression of 1,308 lncRNA transcripts (defined as expression differences $>2$-fold) corresponding to $4.1 \%$ of the studied transcripts. The expression of lncRNAs was successfully validated for upregulated (lnc-BMP2-2, lncCPN2-1, lnc-FZD1-2, lnc-ITPR2-3, Inc-SLC30A4-1, lncSPAM1-6), downregulated (lnc-ACACA-1, lnc-FOXG1-2, lnc-LCP2-2, lnc-RP3-368B9, lnc-TTC34-3), and unregulated (lnc-ERCC5-1, lnc-RP11-480I12.4.1-1) transcripts using qPCR in a cohort of 55 ccRCC and 52 normal renal specimen, thus the specificity of the microarray results is confirmed. Two recent studies investigated the lncRNA expression profile of RCC tissues using microarray technologies, but samples sizes were small $(n=4$ [5]; $n=6[6]$; $n=11$ [7]), thereby limiting powerful statistical analysis; furthermore, the analyses were limited to a set of 984 lncRNAs in the Fachel study [7]. During later courses of our study, Malouf et al. [8] re-analyzed the data obtained in The Cancer Genome Atlas project and identified 1,934 lncRNA expressed in RCC. The catalog of the differentially expressed lncRNAs varied in the profiling studies, but notably, all studies demonstrated a tendency towards an increased ratio of down- to upregulated lncRNAs [6,7]. Earlier studies linked aberrant expression of individual lncRNAs to RCC in small-scaled studies $(n \leq 12$; i.e. GAS5, also termed SERPINC1 [8,14]; aHIF [15]; MALAT1 [8]); we could not confirm GAS5 downregulation in our microarray study; aHIF and MALAT1 was not deregulated.

lncRNAs could serve as diagnostic and prognostic biomarkers: The distinct overexpression of lncRNAs in RCC could be used for the development of a non-invasive diagnostic biomarker. We demonstrated distinct ( $>10$-fold) expression level differences for a number of lncRNAs including lnc-ACACA-1, lnc-BMP2-2, lnc-CPN2-1, lncFOXG1-2, lnc-FZD1-2, lnc-ITPR2-3, lnc-RP3-368B9, lnc-SLC30A4-1, lnc-SPAM1-6, and lnc-TTC34-3; if expression differences are validated by independent researchers, these lncRNAs could represent diagnostic biomarkers. IncRNAs are detectable in bodily fluids and may thereby serve for cancer diagnosis: The PROGENSA ${ }^{\circ}$ PCA3 Assay utilizes the detection of the lncRNA PCA3 in urine as diagnostic biomarker for prostate cancer [16]. Furthermore, plasma levels of HULC were increased in hepatocellular carcinoma [17], H19 in gastric cancer [18], and MALAT-1 in prostate cancer [19] patients. 

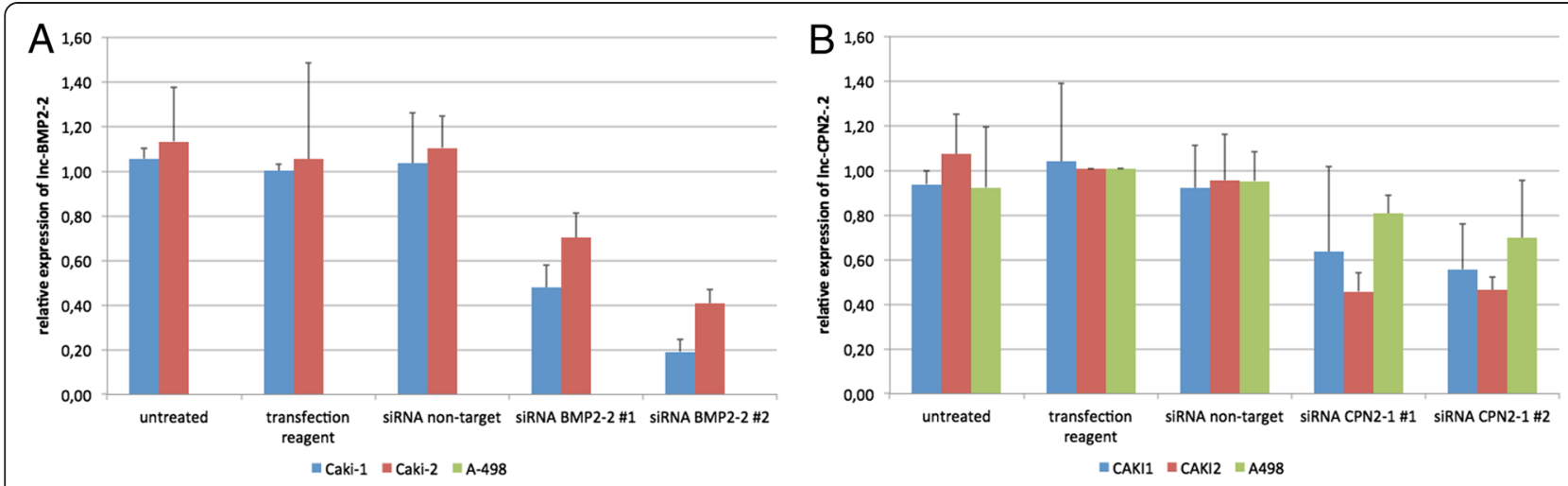

Figure 3 IncRNA expression after siRNA-mediated IncRNA knockdown. Expression of Inc-BMP2-2 (A) and Inc-CPN2-1 (B) was manipulated using specific siRNA in Caki-1, Caki-2, and A-498 renal cell carcinoma cell lines. A-498 did not express Inc-BMP2-2. Inc-BMP2-2 expression was significantly $(p<0.05)$ decreased in siRNA-treated Caki-1 and Caki-2 cells; Inc-CPN2-1 was also decreased in Caki-2 cells.

In addition, lncRNAs may be of prognostic relevance: Fachel et al. [7] reported a 26-gene lncRNA expression profile, but not a single lncRNA, associated with the survival of ccRCC patients. However, the small sample size $(n=16)$ and the lack of a validation cohort limit the informative value of the study [7]. Malouf et al. [8] identified four lncRNA expression clusters among the study cohort, and the survival was significantly reduced in one subgroup (C2). We did not observe a correlation of lncRNAs (used in the validation study) with any clinicopathological parameter nor survival data. Reasons for the failure to identify prognostic relevant lncRNAs include: $(i)$ a microarray study not powered for the identification of expression differences in localized $(n=5)$ and advanced RCC ( $n=10)$, (ii) the selection of lncRNAs for validation based on the differential expression of tumor and normal tissue samples, and (iii) the high number of patients with localized ccRCC (56\% AJCC stage 1 or 2) in the validation cohort. We expect that future studies designed to identify prognostic relevant lncRNAs in RCC patients will be able to present some candidates, because altered expression of specific lncRNAs was predictive of cancer-specific survival in former studies on other human malignancies; i.e. upregulation of HOTAIR in breast cancer [20] and SChLAP1 in prostate cancer patients [21] was associated with a poor prognosis.

As interest in IncRNA research has increased a short time ago, the function of lncRNAs remains largely unknown, but involvement in many fundamental cellular processes is assumed [13]. The short half-life time $(<2 \mathrm{~h})$ of nuclear lncRNA predisposes for regulative functions $[22,23]$. We performed in silico analyses to point out putative functions of dysregulated lncRNAs: $(i)$ lncRNA may act as miRNA sponge (e.g. miR-372 expression is downregulated by the IncRNA HULC [24]). However, the expression levels of several FOXG1-2 target
microRNAs were not correlated to the level of FOXG12 , thus we cannot confirm such a role for the investigated lncRNAs. (ii) Furthermore, lncRNA may function via lncRNA-protein interactions [25]. Using catRAPID omcis [11], we identified putative 91 lncRNA-protein interactions for the dysregulated IncRNAs (lnc-ACACA-1, lnc-BMP2-2, lnc-CPN2-1, lnc-FOXG1-2, lnc-FZD1-2, lncITPR2-3, lnc-LCP2-2, lnc-RP3-368B9, lnc-SLC30A4-1, lnc-SPAM1-6, lnc-TTC34-3). Most of these proteins are functioning in splicing, binding, transport, localization, transcription, translation, and processing of RNA. Providing experimental evidence beyond in silico predictions is necessary in future studies.

Somewhat surprisingly, neither siRNA-mediated knockdown of lncRNA-CPN2-2 nor lnc-BMP2-2 resulted in a significant reduction of cell proliferation. However, the lncRNA knockdown was of moderate success (relative expression change in siRNA-treated cell lines 2- to 5-fold, despite of the extensive optimization of siRNA treatment in preliminary experiments), and thus the toxicity of the transfection reagent may inhibit determining the functional relevance of both lncRNAs. Many lncRNAs regulate nuclear events and must therefore be localized in the nucleus [26]. As is known, the knockdown of nuclear RNAs is often difficult [27], and therefore our efforts to determine the functional relevance of lncRNAs failed.

\section{Conclusions}

In summary, our study reveals that dysregulation of approximately $4 \%$ of the lncRNA transcripts occurs in ccRCC, and altered lncRNA expression may modulate fundamental cellular processes. IncRNA profiles allow to accurately distinguish ccRCC and normal renal tissue. Thus, lncRNAs may be used as non-invasive biomarker for RCC patients. 


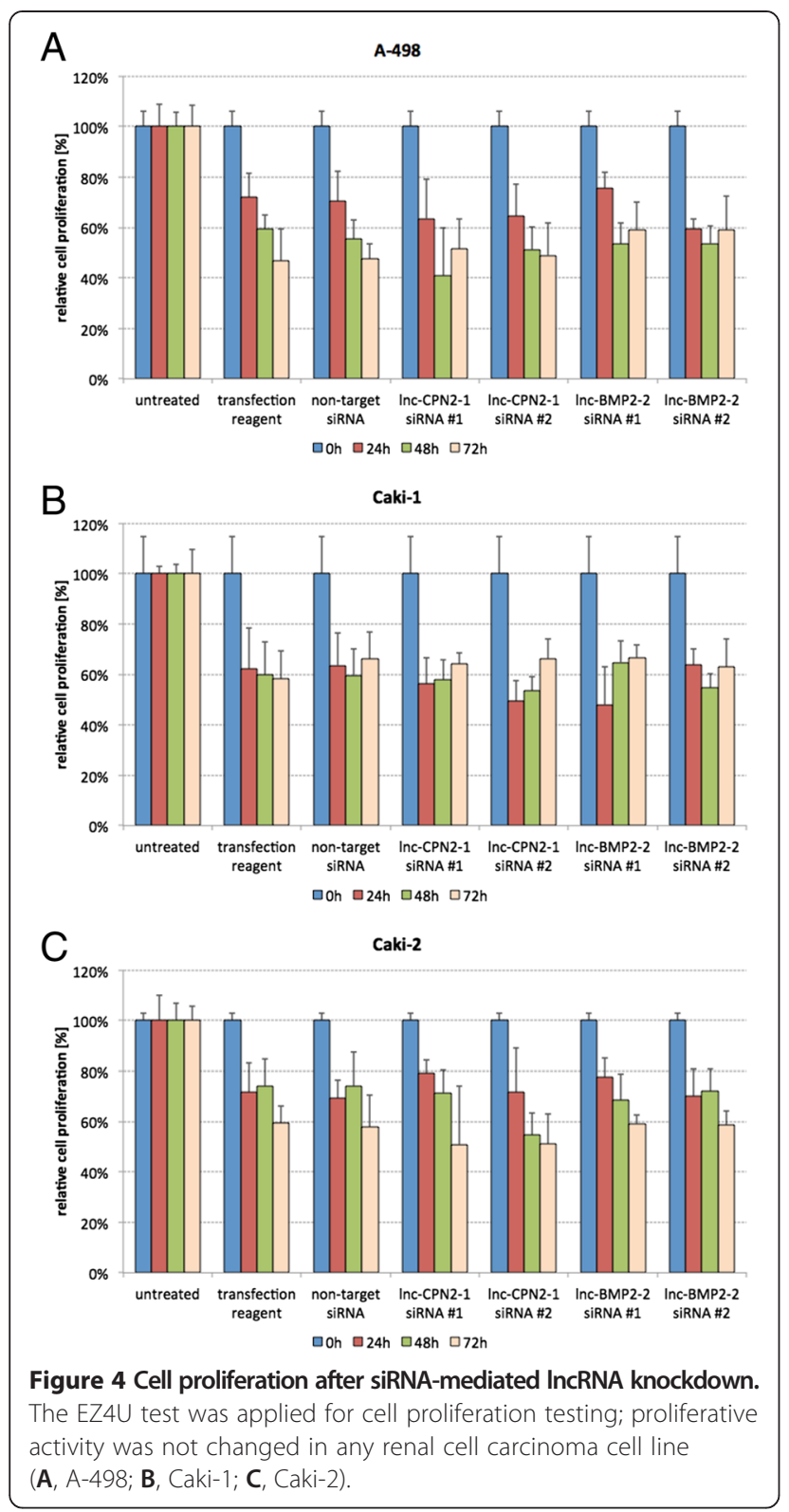

\section{Methods}

\section{Patients}

Biomaterials including fresh-frozen tissues are prospectively collected in the Biobank at the CIO Köln Bonn at the Universitätsklinikum Bonn according to standard operating procedures. Tissue from patients undergoing radical nephrectomy or nephron-sparing surgery was snap frozen, and samples from tumor and normal renal tissue were stored at liquid nitrogen. Hematoxylin- and eosin-stained sections were performed to confirm the histology of the samples. The final histological diagnosis was made on the formalin-fixed paraffin-embedded tissue samples. All cases used in this study were reviewed by an experienced uropathologist; the TNM classification (7th edition from 2009) was applied. We used a discovery cohort with 15 patients (ccRCC and adjacent normal renal tissue) for screening 32,183 lncRNA transcripts; the independent validation cohort consisted of 55 ccRCC and 52 normal renal tissue samples. The detailed clinical-pathological parameters are reported in Table 4. All patients gave written informed consent prior collection of biomaterials. The study was approved by the Ethikkommission at the Universitätsklinikum Bonn (number: 280/12).

\section{Cell culture and siRNA experiments}

The cell lines Caki-1, Caki-2, and A-498 were obtained from the DSMZ (Braunschweig, Germany). All cell lines were maintained at $37^{\circ} \mathrm{C}, 5 \% \mathrm{CO}_{2}$ in RPMI 1640 culture medium, supplemented with $10 \%$ heat-inactivated fetal calf serum and $2 \mathrm{mM}$ of glutamine (PAA, Pasching, Austria). The knockdown of lnc-BMP2-2 (\#1: sense GCG-UUU-UAA-UGU-CCA-CCA-Att, antisense UUGGUG-GAC-AUU-AAA-ACG-Caa; \#2: sense GAA-AGAGAC-UGA-AUA-AUU-Att, antisense UAA-UUA-UUCAGU-CUC-UUU-Ctc) and lnc-CPN2-1 (\#1: sense CACUCA-UCU-UUA-AAU-UAG-Att, antisense UCU-AAUUUA-AAG-AUG-AGU-Gat; \#2: sense CAA-UGA-AACAGA-ACA-GAU-Att, antisense UAU-CUG-UUC-UGUUUC-AUU-Gtt) was performed with individually designed Ambion Silencer Select siRNAs (Life Technologies, Foster City, CA, USA); two different siRNAs were designed for each target. In addition, negative controls (transfection reagent without siRNA; Silencer Select Negative Control siRNA \#1) and a positive control (Silencer GAPDH Positive Control siRNA) were used. The cell lines were seeded at $2.5 \times 10^{5}$ cells/well (6-well plate), and a forward transfection using the Screenfect Transfection Kit (Genaxxon, Ulm, Germany) with a siRNA concentration of $20 \mathrm{pmol}$ was performed according to the manufacturer's recommendations. The transfection complex was replaced after $24 \mathrm{~h}$ with RPMI medium and incubated for up to $72 \mathrm{~h}$. siRNA experiments were confirmed in three independent experiments.

\section{Cell proliferation assay}

The cell lines were seeded at a concentration of $1.5 \times 10^{4}$ cells per well into a 96-well plate. After forward transfection (siRNA concentration $1.2 \mathrm{pmol}$ ), the cells were cultured up to $72 \mathrm{~h}$ to determine the proliferative activity after siRNA-mediated knockdown of lnc-BMP2-2 and lncCPN2-1. The EZ4U assay (Biomedica, Vienna, Austria) was used to determine cell viability using a 340 ATTC Spectra Thermo SLT photometer (Crailsheim, Germany) according to the manufacturer's protocol.

\section{RNA isolation}

Fresh-frozen tissue samples (approximately $50 \mathrm{mg}$ ) were cut on liquid nitrogen and homogenized with Yttrium 
Table 3 Computationally predicted functions by IncRNA-protein interactions

\begin{tabular}{|c|c|c|c|c|c|c|c|c|c|c|c|}
\hline Predicted function & Inc-ACACA-1 & Inc-BMP2-2 & Inc-CPN2-1 & Inc-FOXG1-2 & Inc-FZD1-2 & Inc-ITPR2-3 & Inc-LCP2-2 & Inc-RP3-368B9.1.1-1 & Inc-SLC30A4-1 & Inc-SPAM1-6 & Inc-TTC34-3 \\
\hline mRNA processing & $x$ & $x$ & $x$ & $x$ & $x$ & $x$ & $x$ & $x$ & $x$ & $x$ & $x$ \\
\hline mRNA/RNA splicing & $x$ & $x$ & $x$ & $x$ & $x$ & $x$ & $x$ & $x$ & $x$ & $x$ & $x$ \\
\hline mRNA binding & & $x$ & $x$ & $x$ & $x$ & $x$ & $x$ & & $x$ & $x$ & $x$ \\
\hline RNA localization & $x$ & $x$ & & & & $x$ & $x$ & $x$ & $x$ & $x$ & $x$ \\
\hline Ribonucleoprotein complex & & $x$ & & & $x$ & $x$ & $x$ & $x$ & $x$ & & $x$ \\
\hline RNA transport & $x$ & & & & & $x$ & $x$ & $x$ & $x$ & $x$ & $x$ \\
\hline Transcription & $x$ & & & & & $x$ & $x$ & $x$ & $x$ & $x$ & $x$ \\
\hline Posttranscriptional regulation & & $x$ & $x$ & $x$ & $x$ & $x$ & & $x$ & & & \\
\hline mRNA/RNA stabilization & & $x$ & $x$ & $x$ & $x$ & $x$ & & & & & \\
\hline DNA binding & & & & & & $x$ & & & & $x$ & $x$ \\
\hline Translation & & & & & $x$ & & $x$ & & $x$ & & \\
\hline Protein localization & & & & & & & & & $x$ & & \\
\hline rRNA processing & & & & & & & & & $x$ & & \\
\hline Viral genome expression & & & & & & & & & $x$ & & \\
\hline
\end{tabular}


Table 4 Summary of clinicopathological parameters of the study cohort

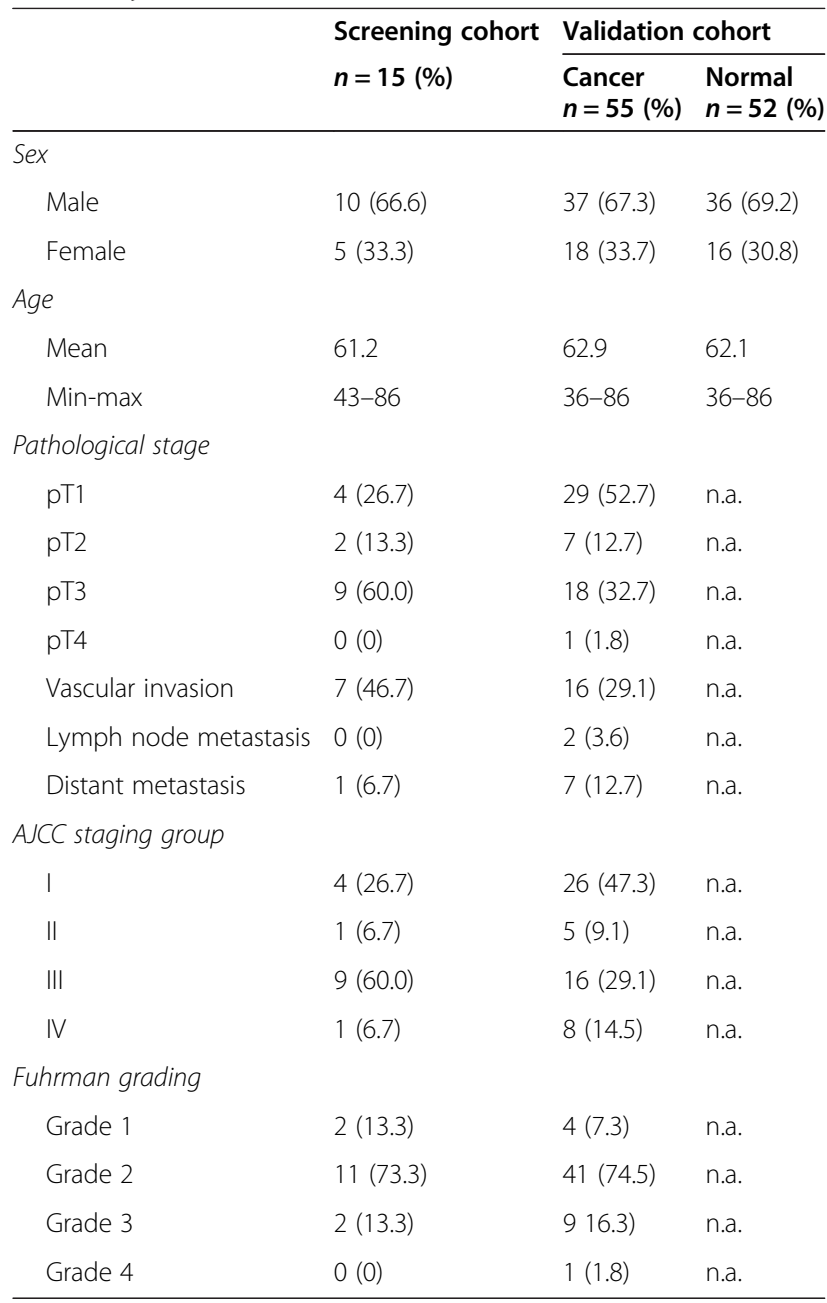

n.a. not applicable.

stabilized Zirkonoxid-Beads (Silibeads, Sigmund-Lindner, Warmensteinach, Germany) using a Precellys ${ }^{\circ} 24$ tissue homogenizer (Peglab, Erlangen, Germany). Total RNA was then isolated using the mirVana miRNA Isolation Kit (Ambion, Foster City, CA, USA) according to the manufacturer's recommendation. To remove residual DNA fragments, we treated the isolate twice with DNase (DNAfree Kit, Ambion). RNA quantity was determined with a NanoDrop 2000 spectrophotometer (Thermo Scientific, Wilmington, DE, USA). RNA integrity was measured in samples used for microarray experiments using the Bioanalyzer 2100 with a RNA 6000 Nano Kit (Agilent Technologies, Santa Clara, CA, USA); only samples with a RIN $>6$ were used. RNA degradation was excluded by agarose gel electrophoresis in samples used for PCR.

\section{Microarray}

The microarray experiments were performed by Biogazelle (Zwijnaarde, Belgium) as a contract service. RNA isolates from 15 corresponding normal renal and ccRCC tissues (100 ng total RNA) were sent on dry ice to Biogazelle. A custom microarray (Agilent SurePrint G3 technology) based on LNCipedia 2.1 [10] was used to study 32,183 lncRNA transcripts belonging to 17,512 lncRNAs. Background substracted and normalized (log2-based) expression data were provided from Biogazelle for further data analysis. Expression data of lncRNAs for ccRCC and normal tissues was groupwise normalized deducting group mean value. Pairwise fold change, for each gene, was calculated by dividing ccRCC group mean of RNA expression level by normal group mean of expression levels. Pearson's correlation coefficient was used for correlation analyses (R-Base Package v3.0.2). A heatmap was created using gplots v2.12.1 and RColorBrewer v1.0-5.

\section{Real-time PCR}

Quantitative real-time PCR was performed to validate the microarray experiments using an independent cohort of $55 \mathrm{ccRCC}$ and 52 normal renal tissue samples and to confirm the success of siRNA-mediated lncRNA knockdown. Complementary DNA (cDNA) was synthesized with $1 \mu \mathrm{g}$ RNA using the PrimeScript RT Reagent Kit with gDNA Eraser. Real-time PCR was performed with 5 ng cDNA template using the $1 \times$ SYBR Premix Ex Taq II with ROX Plus and $10 \mathrm{pmol} / \mu \mathrm{l}$ PCR primers (see Additional file 3: Table S1 for primer sequences); all reagents were from Takara Bio, Saint-Germain-en-Laye, France. PCR was performed using an ABIPrism 7900 HT Fast Real-Time PCR System (Applied Biosystems, Foster City, CA, USA). Data analysis was performed using Qbase + (Biogazelle) with PPIA and ACTB as reference genes in the 2- $\Delta \Delta C T$ algorithm. SPSS Statistics v21 (IBM, Ehningen, Germany) was used for statistical analyses (Mann-Whitney- $U$ test, Cox regression analysis).

\section{MicroRNA quantification}

Quantitative real-time PCR was used to determine the tissue levels of several FOXG1-2 microRNA targets in each ten normal and malignant renal tissues using the Qiagen miScript SYBR Green PCR technology (Hilden, Germany): 1,000 ng total RNA was reverse transcribed and real-time PCR was performed with $5 \mathrm{ng}$ cDNA template. Pre-designed miScript Primer Assays for miR-519a, miR-519b, miR-519c, miR-548, miR-3120, miR-4284, miR4658 , and SNORD43 were used. PCR was performed using the ABIPrism 7900 HT Fast Real-Time PCR System. Data analysis was performed using Qbase + with SNORD43 as reference genes in the 2- $\Delta \Delta C$ T algorithm. IBM SPSS Statistics v21 was used for statistical analyses (Pearson correlation test). 


\section{In silico analyses}

The MiRTarget2 algorithm [9] was used to predict microRNA seeds within the validated lncRNA transcripts. Potential lncRNA-protein interactions were determined using the catRAPID omics algorithm [11]. The cellular functions of proteins were retrieved with GeneMANIA [12]. Standard settings were used in all software packages.

\section{Availability of supporting data}

The microarray data sets supporting the results of this article are available in the Gene Expression Omnibus repository (data entry: GSE61763).

\section{Additional files}

Additional file 1: Table S2. Summary of predicted IncRNA-protein interactions (catRAPID omics algorithm).

Additional file 2: Table S3. Summary of IncRNA interacting networks for cellular functions (GeneMANIA algorithm).

Additional file 3: Table S1. List of PCR primers.

\section{Competing interests}

The authors declare that they have no competing interests.

\section{Authors' contributions}

JJCB and DS carried out the InCRNA and microRNA expression analyses. MD performed the statistical analysis of microarray data. DS, IS, and SS performed the siRNA experiments. JJCB and JE performed the statistics and drafted the manuscript. SP and SCM participated in the study design and helped to draft the manuscript. All authors read and approved the final manuscript.

\section{Acknowledgements}

The collection of tissue samples was performed within the framework of the Biobank of the Center for Integrated Oncology Köln Bonn.

\section{Author details}

${ }^{1}$ Department of Urology, University Hospital Bonn, Bonn, Germany. ${ }^{2}$ Institute of Pathology, University Hospital Bonn, Bonn, Germany. ${ }^{3}$ Department of Prostate Cancer Research, University Hospital Bonn, Bonn, Germany. ${ }^{4}$ Center of Integrated Oncology, University Hospital Bonn, Bonn, Germany.

Received: 15 October 2014 Accepted: 15 January 2015

Published online: 08 February 2015

\section{References}

1. Chow WH, Dong LM, Devesa SS. Epidemiology and risk factors for kidney cancer. Nat Rev Urol. 2010;7:245-57.

2. Dietrich D, Meller S, Uhl B, Ralla B, Stephan C, Jung K, et al. Nucleic acid-based tissue biomarkers of urologic malignancies. Crit Rev Clin Lab Sci. 2014:1-27.

3. Geisler S, Coller J. RNA in unexpected places: long non-coding RNA functions in diverse cellular contexts. Nat Rev Mol Cell Biol. 2013;14:699-712.

4. Gibb EA, Vucic EA, Enfield KS, Stewart GL, Lonergan KM, Kennett JY, et al. Human cancer long non-coding RNA transcriptomes. PLoS One. 2011;6:e25915.

5. Qin C, Han Z, Qian J, Bao M, Li P, Ju X, et al. Expression pattern of long non-coding RNAs in renal cell carcinoma revealed by microarray. PLOS One. 2014:9:e99372.

6. Yu G, Yao W, Wang J, Ma X, Xiao W, Li H, et al. IncRNAs expression signatures of renal clear cell carcinoma revealed by microarray. PLoS One. 2012; 7:e42377.

7. Fachel AA, Tahira AC, Vilella-Arias SA, Maracaja-Coutinho V, Gimba ER, Vignal $\mathrm{GM}$, et al. Expression analysis and in silico characterization of intronic long noncoding RNAs in renal cell carcinoma: emerging functional associations. Mol Cancer. 2013;12:140.
8. Malouf GG, Zhang J, Yuan Y, Comperat E, Roupret M, Cussenot O, et al. Characterization of long non-coding RNA transcriptome in clear-cell renal cell carcinoma by next-generation deep sequencing. Mol Oncol. 2014.

9. Wang $X$, El Naqa IM. Prediction of both conserved and nonconserved microRNA targets in animals. Bioinformatics (Oxford, England). 2008;24:325-32.

10. Volders PJ, Helsens K, Wang X, Menten B, Martens L, Gevaert K, et al. LNCipedia: a database for annotated human IncRNA transcript sequences and structures. Nucleic Acids Res. 2013;41:D246-51.

11. Agostini F, Zanzoni A, Klus P, Marchese D, Cirillo D, Tartaglia GG. catRAPID omics: a web server for large-scale prediction of protein-RNA interactions. Bioinformatics (Oxford, England). 2013;29:2928-30.

12. Warde-Farley D, Donaldson SL, Comes O, Zuberi K, Badrawi R, Chao P, et al The GeneMANIA prediction server: biological network integration for gene prioritization and predicting gene function. Nucleic Acids Res. 2010;38: W214-20.

13. Martens-Uzunova ES, Bottcher R, Croce CM, Jenster G, Visakorpi T, Calin GA Long noncoding RNA in prostate, bladder, and kidney cancer. Eur Urol. 2013;65:1140-51.

14. Qiao HP, Gao WS, Huo JX, Yang ZS. Long non-coding RNA GAS5 functions as a tumor suppressor in renal cell carcinoma. Asian Pac J Cancer Prev. 2013;14:1077-82.

15. Thrash-Bingham CA, Tartof KD. aHIF: a natural antisense transcript overexpressed in human renal cancer and during hypoxia. J Natl Cancer Inst. 1999:91:143-51.

16. Bradley LA, Palomaki GE, Gutman S, Samson D, Aronson N. Comparative effectiveness review: prostate cancer antigen 3 testing for the diagnosis and management of prostate cancer. J Urol. 2013;190:389-98.

17. Xie H, Ma H, Zhou D. Plasma HULC as a promising novel biomarker for the detection of hepatocellular carcinoma. BioMed Res Int. 2013;2013:136106.

18. Arita T, Ichikawa D, Konishi H, Komatsu S, Shiozaki A, Shoda K, et al. Circulating long non-coding RNAs in plasma of patients with gastric cancer. Anticancer Res. 2013:33:3185-93.

19. Ren S, Wang F, Shen J, Sun Y, Xu W, Lu J, et al. Long non-coding RNA metastasis associated in lung adenocarcinoma transcript 1 derived miniRNA as a novel plasma-based biomarker for diagnosing prostate cancer. Eur J Cancer (Oxford, England: 1990). 2013;49:2949-59.

20. Gupta RA, Shah N, Wang KC, Kim J, Horlings HM, Wong DJ, et al. Long non-coding RNA HOTAIR reprograms chromatin state to promote cancer metastasis. Nature. 2010;464:1071-6.

21. Prensner JR, lyer MK, Sahu A, Asangani IA, Cao Q, Patel L, et al. The long noncoding RNA SChLAP1 promotes aggressive prostate cancer and antagonizes the SWI/SNF complex. Nat Genet. 2013:45:1392-8.

22. Tani H, Mizutani R, Salam KA, Tano K, ljiri K, Wakamatsu A, et al. Genome-wide determination of RNA stability reveals hundreds of short-lived noncoding transcripts in mammals. Genome Res. 2012;22:947-56.

23. Clark MB, Johnston RL, Inostroza-Ponta M, Fox AH, Fortini E, Moscato P, et al. Genome-wide analysis of long noncoding RNA stability. Genome Res. 2012:22:885-98.

24. Wang J, Liu X, Wu H, Ni P, Gu Z, Qiao Y, et al. CREB up-regulates long non-coding RNA, HULC expression through interaction with microRNA-372 in liver cancer. Nucleic Acids Res. 2010;38:5366-83.

25. Zhu J, Fu H, Wu Y, Zheng X. Function of IncRNAs and approaches to IncRNA-protein interactions. Sci China Life Sci. 2013;56:876-85.

26. Zhang B, Gunawardane L, Niazi F, Jahanbani F, Chen X, Valadkhan S. A novel RNA motif mediates the strict nuclear localization of a long noncoding RNA. Mol Cell Biol. 2014;34:2318-29.

27. Ploner A, Ploner C, Lukasser M, Niederegger $\mathrm{H}$, Huttenhofer $\mathrm{A}$. Methodological obstacles in knocking down small noncoding RNAs. RNA (New York, NY). 2009:15:1797-804. 